\title{
Posttraumatisches Wachstum
}

\author{
Judith Mangelsdorf
}

Online publiziert: 29. Januar 2020

(C) Der/die Autor(en) 2020

Zusammenfassung Das Phänomen des posttraumatischen Wachstums beschreibt die Möglichkeit, dass Opfer traumatischer Erfahrung nicht nur psychische und soziale Einbußen als Folge des Erlebten erfahren, sondern auch persönliche Entwicklungsprozesse angestoßen werden können. Dieser Artikel der Zeitschrift für Psychodrama und Soziometrie gibt einen Überblick über den aktuellen Wissensstand zu posttraumatischem Wachstum sowie dessen Entstehung und Förderung. Es wird der Zusammenhang zu anderen Reaktionen auf traumatische Ereignisse, wie Resilienz und posttraumatische Belastungsstörung, erläutert. Posttraumatisches Wachstum kann zu einer zentralen Ressource im Leben von Betroffenen werden und damit konzeptionell eine wichtige Bereicherung für die Arbeit mit traumatisierten Klientinnen und Klienten sein.

Schlüsselwörter Posttraumatisches Wachstum · Resilienz $\cdot$ Posttraumatische Belastungsstörung $\cdot$ Lebensereignisse $\cdot$ Life-Events $\cdot$ Psychodrama

Psychotraumatologie $\cdot$ Traumatherapie 


\section{Posttraumatic growth}

Abstract The term posttraumatic growth describes the phenomenon of personal growth after adverse life events. It highlights the possibility that potentially traumatic experiences cannot only cause suffering but can also foster personal growth. This article in the journal Zeitschrift für Psychodrama und Soziometrie provides an overview of the latest research and practice in this field, as well its origin and facilitation. It highlights the relation to other reactions to trauma, including resilience, recovery, and posttraumatic stress disorder. Posttraumatic growth can be a critical resource in the life of people who have experienced great adversity and conceptually be an important enrichment for the clinical work with traumatizes individuals.

Keywords Posttraumatic growth · Resilience $\cdot$ PTSD $\cdot$ Life events · Psychodrama · Psychotraumatology $\cdot$ Trauma therapy

\section{Einführung}

„Was uns nicht umbringt, macht uns stark.“ Diese weitverbreitete Redewendung, die auf den deutschen Philosophen Friedrich Nietzsche (1997) zurückgeht, beschreibt ein psychologisches Phänomen, das in jüngster Zeit vermehrt wissenschaftliche Aufmerksamkeit gewonnen hat: Das posttraumatische Wachstum. Doch anders als es Nietzsches Zitat suggeriert, führt eine kritische Erfahrung, die nicht das eigene Leben kostet, nicht zwangsweise zu Wachstum und Stärke. Was genau posttraumatisches Wachstum ist, unter welchen Umständen es auftritt und wie es gefördert werden kann, steht deshalb im Zentrum dieses Artikels.

\subsection{Traumatische Erfahrungen}

Zum Leben jedes Menschen gehören einschneidende Ereignisse. Hierzu zählen normative Erfahrungen wie die Geburt eines Kindes oder der Tod älterer Angehöriger. Gleichzeitig konfrontiert das Leben viele Menschen mit schwerwiegenden, nicht normativen Ereignissen wie Gewalterfahrungen, lebensbedrohlichen Unfällen oder sexuellen Übergriffen. Diese als potenziell traumatisch beschriebenen Ereignisse können im kritischsten Fall in eine posttraumatische Belastungsstörung (PTBS) münden.

Im Kontext des posttraumatischen Wachstums ist es jedoch wesentlich, den Traumabegriff zu erweitern. Auch einschneidende Ereignisse, die nicht unmittelbar mit einer Bedrohung des eigenen Lebens in Zusammenhang stehen, erfordern häufig massive Anpassungsleistungen und können zu posttraumatischem Wachstum führen. Hierzu zählt beispielsweise das plötzliche Verlassenwerden durch einen langjährigen Partner.

Die folgenden Ausführungen beziehen sich gleichermaßen auf potenziell traumatische Ereignisse wie auch auf einschneidende Erlebnisse im nicht-klinischen Sinne, da beide Auslöser für posttraumatisches Wachstum sein können. 


\subsection{Posttraumatisches Wachstum - eine Begriffsklärung}

„Wie hat die traumatische Erfahrung Ihr Leben verändert?" Die Antwort auf diese Frage ist oft vielschichtig und nicht selten von schweren Verlusten geprägt. Der Verlust bedeutender Beziehungen wie beispielsweise die zum verstorbenen Partner, aber auch der Verlust von Gesundheit oder sexueller Integrität nach Übergriffen, sind nur exemplarisch für die Vielzahl an erlebten Beeinträchtigungen. Umso interessanter ist eine Beobachtung, die verschiedene Wissenschaftler bereits in den 1990er-Jahren machten. Die Forscherteams stießen vermehrt auf Berichte, in denen die befragten Personen schilderten, dass die traumatische Erfahrung sie nicht nur viel gekostet, sondern auch bereichert hatte. Überlebende unterschiedlichster Ereignisse teilten die Wahrnehmung, dass sie durch die Auseinandersetzung mit dem Erlebten ein tieferes Verständnis von sich selbst und dem Leben im Ganzen gewonnen hatten. Dieses Phänomen der traumaassoziierten Erweiterung psychischer Ressourcen nannten Tedeschi und Calhoun (1996, S. 455) posttraumatisches Wachstum, während Park et al. (1996, S. 71) es als stress-bezogenes Wachstum (stress-related growth) bezeichneten. Tedeschi und Calhoun (1996, S. 455) identifizierten fünf spezifische Areale posttraumatischen Wachstums: eine größere Wertschätzung dem Leben gegenüber, tiefere soziale Beziehungen, mehr persönliche Stärke, neue Prioritäten im Leben und einen erweiterten Sinn für Spiritualität (Tedeschi und Calhoun 1996, 2004). Die Integration der Wachstumsperspektive erweiterte die wissenschaftliche Auseinandersetzung mit den Folgen kritischer Erfahrungen. Sowohl die Resilienzforschung als auch die Erforschung von PTBS fokussierten auf die Frage, wann es Betroffenen gelingt, keine negativen Konsequenzen zu erleiden oder diese nach dem Auftreten zu bewältigen (vgl. Bonanno 2004). Das Konzept des posttraumatischen Wachstums ergänzte diese Perspektive um die Möglichkeit, dass Betroffene auch positive Auswirkungen nach der erfolgreichen Bewältigung von Traumata erleben können.

\section{Entstehung und Voraussetzungen}

Die Forschung hat gezeigt, dass einige Menschen nach potenziell traumatischen Erfahrungen von posttraumatischem Wachstum berichten, während andere unberührt bleiben oder vorwiegend negative Konsequenzen erleben. Es stellt sich daher die Frage, unter welchen Bedingungen posttraumatisches Wachstum konkret entsteht. Die am besten beforschte Entstehungstheorie vermutet die Erschütterung persönlicher Grundüberzeugungen (core belief disruption; Cann et al. 2010, S. 19) als wichtigste Voraussetzung für posttraumatisches Wachstum. Cann und Kollegen (ebd.) nehmen an, dass es im Zuge der Konfrontation mit potenziell traumatischen Erfahrungen zu einer schweren Erschütterung des eigenen Weltbildes kommen muss, damit danach Wachstum entstehen kann. Vergleichbar mit einem Erdbeben, dass das vorhandene Stadtbild zu großen Teilen zerstört und einen Neu- oder Wiederaufbau notwendig macht, wird davon ausgegangen, dass die psychische Erschütterung offenbar notwendige Voraussetzung für weitreichende Veränderungsprozesse ist.

Seit den ersten frühen Forschungsarbeiten entwickelten sich neben dieser Annahme eine Vielzahl von Modellen, die zum Ziel hatten, das Auftreten posttraumatischen 
Wachstums zu erklären (Jayawickreme und Blackie 2014, 2016; Joseph und Linley 2004; Park et al. 1996; Park und Helgeson 2006; Tedeschi und Calhoun 1996, 2004). Eine der weitreichendsten unter ihnen ist die Theorie dynamischer Systeme (Dynamic systems framework; Cicchetti und Toth 2009; Masten 2014; Overton 2015). Sie basiert auf der Annahme, dass menschliche Entwicklung im Allgemeinen sowohl durch die Interaktion verschiedenster molekularer, psychischer und sozialer Systeme geprägt ist als auch durch Einflüsse der Umwelt. Bei der Konfrontation mit einem potenziell traumatischen Ereignis kann es zu einer Störung oder Destabilisierung von Systemfunktionen kommen. Bisherige Rollen, Denk- und Verhaltensmuster sind durch die veränderte Situation und Erfahrungswelt nicht mehr aufrechtzuerhalten. Aus einer psychodramatischen Perspektive bedeutet dies auch, dass für die Identität bedeutsame Rollen und Rollenzuschreibungen in Frage gestellt oder gänzlich zerstört werden. Ein klassisches Beispiel hierfür liegt im plötzlichen Tod eines Lebenspartners, dessen Ableben nicht nur einen starken emotionalen Verlust verursacht, sondern auch weitreichende systemische Einbußen mit sich bringt. Nach der Theorie dynamischer Systeme kann die durch das traumatische Ereignis hervorgerufene Destabilisierung zu einer Reorganisation, Veränderung oder Transformation bestehender Systemebenen führen und damit auch zu Wachstum (Mangelsdorf et al. 2019, S. 2). So kann beispielsweise eine Folge des Verlustes des Lebenspartners sein, dass soziale Beziehungen zu Familienmitgliedern, Freunden oder auch zu fremden Personen neu entstehen, sich intensivieren oder in ihrer Qualität verändern. Zusammengefasst kann posttraumatisches Wachstum damit als das Potenzial eines dynamischen Systems gesehen werden, sich an aversive Ereignisse anzupassen und dadurch die vorherigen Ressourcen zu erweitern. Auf einem individuellen Level können diese Ressourcen psychische Komponenten wie beispielsweise das Wissen um den eigenen Lebenssinn beinhalten, aber auch soziale Aspekte wie etwa die Tiefe von Beziehungen.

\section{Resilienz und posttraumatisches Wachstum}

Anders als die Redewendung „Was uns nicht umbringt, macht uns stark“ nahelegt, ist posttraumatisches Wachstum nur eines der möglichen Reaktionsmuster nach traumatischen Ereignissen. Es lassen sich insgesamt vier Reaktionsformen unterscheiden: Resilienz, Erholung, posttraumatische Belastungsstörung und posttraumatisches Wachstum (Abb. 1; in Anlehnung an Mangelsdorf 2014). Die vier Graphen verdeutlichen wie stark und andauernd die zunächst erlebte psychische Beeinträchtigung durch das Ereignis in der jeweiligen Reaktionsform ist und ob die Adaptationsphase in ein erhöhtes psychisches Funktionsniveau der Betroffenen mündet.

Resilienz Die resiliente Reaktion ist dadurch gekennzeichnet, dass es nur zu einem geringen und kurzzeitigen Abfall der psychischen Funktionalität kommt. Betroffene erreichen innerhalb kurzer Zeit wieder ihr psychisches Ausgangsniveau und können ihr Leben wie bislang fortsetzen. 

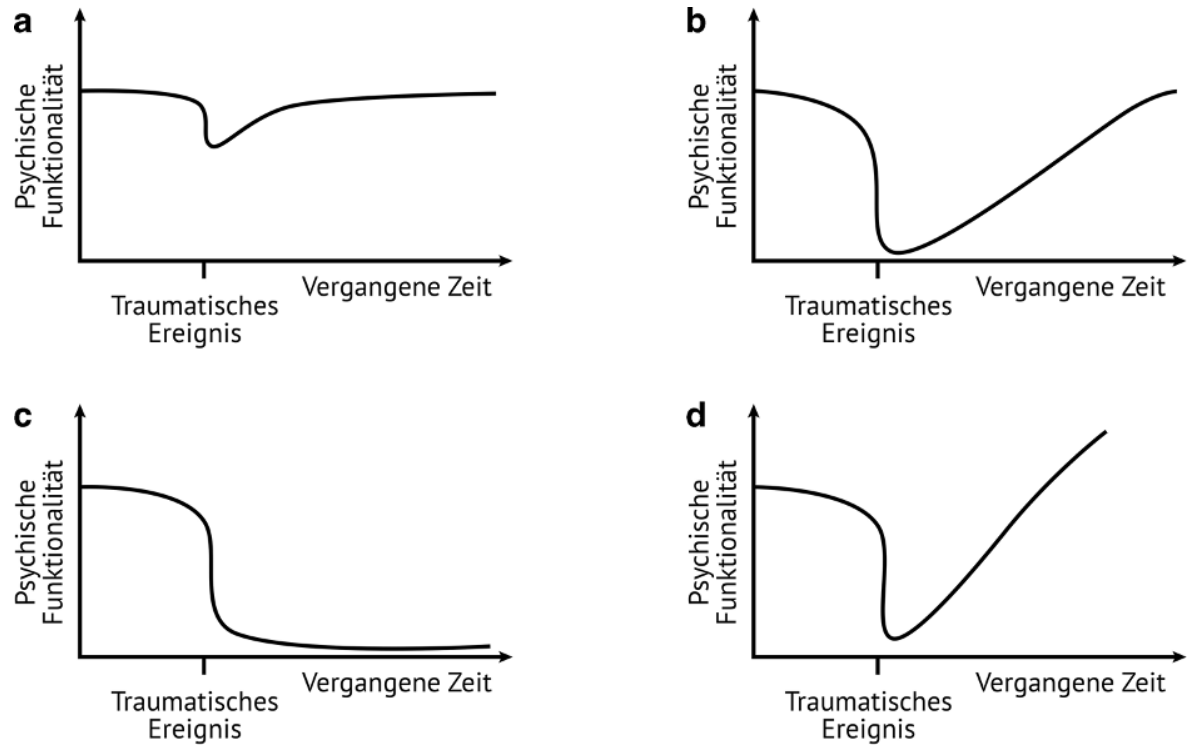

Abb. 1 Psychoemotionale Reaktionsformen nach traumatischen Erfahrungen. a Resilienz, b Erholung, c Posttraumatische Belastungsstörung, d Posttraumatisches Wachstum

Erholung In Abgrenzung dazu erleben Betroffene im Fall der Erholung erhebliche psychische Einbußen, die sich über einen längeren Zeitraum erstrecken können. Hier kommt es zu der oben beschriebenen psychischen Erschütterung, die mit der Zeit kompensiert wird, sodass Menschen, die dieses Reaktionsmuster zeigen, wieder ihr Ausgangsniveau erreichen. In Abgrenzung zum posttraumatischen Wachstum kommt es jedoch nicht zu einer Erweiterung der psychischen Ressourcen.

Posttraumatische Belastungsstörung Die wohl am intensivsten studierte Reaktion auf traumatische Erfahrungen ist die der posttraumatischen Belastungsstörung. In diesem Fall kommt es zu einem starken Abfall des psychischen Funktionsniveaus, der entweder unmittelbar auf das Ereignis folgt oder auch zeitversetzt eintreten kann. In vielen Fällen gelingt es Betroffenen nur mit professioneller Hilfe, das volle $\mathrm{Maß}$ an psychischer Funktionalität wiederherzustellen. Die erlebten Einbußen sind häufig massiv und langanhaltend. Nicht selten erleben sie lebenslange psychische Einschränkungen, die auf das traumatische Ereignis zurückzuführen sind.

Posttraumatisches Wachstum Das letzte psychische Reaktionsmuster besteht im posttraumatischen Wachstum. Auch hier folgen auf die traumatische Erfahrung zunächst starke psychische Einbußen. Ähnlich wie im Fall der posttraumatischen Belastungsstörung und des Erholungsmusters kämpfen Betroffene zunächst mit der Anpassung an die veränderte psycho-emotionale Situation. Anders jedoch als in diesen Fällen, führt die Auseinandersetzung mit der potenziell traumatischen Erfahrung nicht nur zu einer Adaptation zurück zum ursprünglichen Funktionsniveau, sondern zu einer Erweiterung der psychischen Ressourcen. Wichtig zu betonen ist, 
dass sich posttraumatische Belastungsstörung (PTBS) und posttraumatisches Wachstum als Reaktionen gegenseitig nicht ausschließen, sondern häufig in einem engen Zusammenhang stehen. So kann beispielsweise posttraumatisches Wachstum der Bewältigung einer PTBS folgen. Ob es gelingt, die Erfahrung eines potenziell traumatischen Ereignisses in Wachstum münden zu lassen, hängt von verschiedenen Faktoren ab, auf die weiter unten näher eingegangen wird.

Eines der häufigsten Missverständnisse ist die Gleichsetzung von posttraumatischem Wachstum und Resilienz. Während beide anstrebenswerte psychische Reaktionsmuster darstellen, handelt es sich um vollständig distinkte Phänomene, die nach jetzigem Forschungsstand kaum zur selben Zeit in Bezug auf das gleiche Ereignis auftreten. Im Zentrum der Entstehung von posttraumatischem Wachstum steht die Erschütterung psychischer Grundannahmen. Diese Destabilisierung des bestehenden psychischen Systems hat zunächst häufig die Konsequenz, dass das Wohlbefinden und die psychische Funktionalität erheblich leiden. Statt einer resilienten Reaktion, die ja gerade dadurch charakterisiert ist, vom Ereignis nicht oder nur in geringem Maße und kurzfristig beeinträchtigt zu sein, geht dem posttraumatischen Wachstum oftmals eine Zeit des Leidens voraus. Bei einer resilienten Reaktion, also beim Ausbleiben einer psychischen Erschütterung, kommt es im Gegensatz zum posttraumatischen Wachstum nicht oder nur sehr selten zu einer anschließenden Erweiterung psychischer Ressourcen.

\section{Kann man persönliches Wachstum messen? Eine Herausforderung der Empirie}

Als die Forschergruppen um Richard Tedeschi und Crystal Park sich auf den Weg machten, das Phänomen des posttraumatischen Wachstums wissenschaftlich zu erforschen, gab es keine etablierten reliablen Messinstrumente. Alles, worauf damals zurückgegriffen werden konnte, waren die Aussagen von Betroffenen, die schwere Erkrankungen, Gewalt oder Naturkatastrophen überlebt hatten. Beide Forschungsgruppen erstellten jeweils einen Fragebogen mit dem Ziel, posttraumatisches Wachstum retrospektiv zu messen: den Post-traumatic Growth Inventory (PTGI, Tedeschi und Calhoun 1996) und die Stress Related Growth Scale (SRS, Park et al. 1996). Beide Messinstrumente geben Aussagen positiver Veränderung vor, beispielsweise: „Durch das Ereignis habe ich heute tiefere soziale Beziehungen." Teilnehmende sollen dann angeben, wie sehr sie diesen Aussagen auf einer Skala von 1 bis 5 zustimmen. In den zwei Dekaden, die seither vergangen sind, entwickelten sich der PTGI und die SRS zu den am meisten genutzten Skalen zur Erfassung posttraumatischen Wachstums. Viele hunderte Studien, nicht nur der psychologischen, sondern auch der medizinischen Forschung, griffen auf sie zurück.

Erst in den letzten Jahren mehrten sich die Stimmen der Wissenschaftler, die die Belastbarkeit von retrospektiven Selbstaussagen nach potenziell traumatischen Ereignissen kritisch betrachten. Jayawickeme und Blackie (2014, S. 316-317; 2016, S. 19-22) führten vor allem folgendes Problem ins Feld: Um die Aussage beantworten zu können, in welchem Maß ich heute tiefere soziale Beziehungen habe als vor einem spezifischen Ereignis, muss ich mir klar machen, wie es heute um meine 
sozialen Beziehungen steht, wie meine Beziehungen vor dem Ereignis waren, wie groß die Differenz zwischen damals und heute ist und wie viel dieser wahrgenommenen Differenz tatsächlich nur auf das eine untersuchte Lebensereignis zurückzuführen ist und nicht auf andere Erfahrungen, die in der Zwischenzeit gesammelt wurden. Zusammengenommen ist diese retrospektive Selbsteinschätzung ein derart komplexer Prozess, dass nicht davon ausgegangen werden kann, dass Befragte reliable Selbstaussagen treffen können. Mit dieser sehr präzisen Einschätzung stellten Jayawickreme und Blackie (2014) fast 20 Jahre der Forschung zu posttraumatischem Wachstum auf den Prüfstand.

Neue methodische Herangehensweisen sollen nun Abhilfe schaffen. Studien, die mit Hilfe von EEG- (Rabe et al. 2006) und MRT-Daten (Mangelsdorf 2017) versuchen, persönliches Wachstum messbarer zu machen, stehen vor der Herausforderung der Multidimensionalität und Komplexität des Phänomens. In der größten bisher veröffentlichten Meta-Analyse zu posttraumatischem Wachstum führten Mangelsdorf et al. (2019) die Daten von 122 längsschnittlichen Studien zusammen, die einschneidende Lebensereignisse untersuchten, um die Echtheit posttraumatischen Wachstums zu hinterfragen. In die Meta-Analyse wurden nur Studien einbezogen, die nicht aktiv durch Interventionen Einfluss nahmen auf die Teilnehmenden. Alle eingeschlossenen Studien erhoben zu mehreren Messzeitpunkten um das kritische Ereignis psychische Dimensionen wie beispielsweise Lebenssinn, Selbstwert oder Spiritualität. Die Autoren fanden heraus, dass nach durchschnittlich eineinhalb Jahren viele Probanden einen höheren Selbstwert, tiefere Beziehungen und eine bessere Alltagsbewältigung besaßen als vor dem Ereignis.

\section{Unterstützende Faktoren für posttraumatisches Wachstum}

Noch immer steckt die längsschnittliche Forschung ${ }^{1} \mathrm{zu}$ posttraumatischem Wachstum in den Kinderschuhen. So beziehen sich die meisten Erkenntnisse über das, was Menschen darin unterstützt, gestärkt aus potenziell traumatischen Ereignissen hervorzugehen, auf Querschnittsstudien und können damit nur als erste Ideengeber gesehen werden. In einer internationalen Vergleichsstudie untersuchten Mangelsdorf und Eid (2015) drei zentrale Faktoren: die Anwesenheit positiver Emotionen, soziale Unterstützung und das Schöpfen von Sinn aus dem Erlebten. Alle drei Faktoren waren hochgradig mit posttraumatischem Wachstum assoziiert. Menschen, denen es gelang, trotz der kritischen Erfahrungen Momente positiver Emotionalität herzustellen, die unterstützende soziale Beziehungen hatten und die für sich im Nachhinein dem Erlebten einen Sinn beimessen konnten, zeigten häufiger posttraumatisches Wachstum. Während die obigen drei Faktoren nicht die einzigen sind, die in der

\footnotetext{
1 Längsschnittliche Studien begleiten die Teilnehmenden an den Untersuchungen über längere Zeit und haben mehrere Messzeitpunkte. Damit ist es möglich, Entwicklungsprozesse und Veränderungen zu untersuchen, wodurch auch kausale Zusammenhänge festgestellt werden können. Querschnittsstudien haben nur einen Messzeitpunkt. Hiermit zeigen sie zwar, welche Faktoren einen Zusammenhang zueinander haben, lassen aber keine kausalen Schlüsse zu.
} 
Literatur vorgestellt werden, können sie als zentrale Leitfaktoren für Interventionen zur Unterstützung posttraumatischen Wachstums verstanden werden.

\section{Möglichkeiten zur Förderung posttraumatischen Wachstums in der Traumatherapie}

Die Mehrheit der traumatherapeutischen Methoden, wie beispielsweise TRIMB (Spangenberg 2015) oder EMDR (Shapiro 2017), zielen darauf, traumaassoziierte Symptome abzubauen und die Erfahrung zu integrieren. Diese Herangehensweise ist auch bei der anschließenden Förderung posttraumatischen Wachstums Grundlage therapeutischen Handelns. Ergänzt wird sie durch den wachstumsorientierten Fokus. Der wichtigste Grundsatz bei der therapeutischen Arbeit an posttraumatischem Wachstum ist die Wahl des richtigen Zeitpunkts. Posttraumatisches Wachstum ist nicht Folge der potenziell traumatischen Erfahrung selbst, sondern der Bewältigung dieser Erfahrung (Tedeschi und Calhoun 2004, S. 1). Wie aus dem Verlaufsmodell $\mathrm{zu}$ posttraumatischem Wachstum in Abb. 1 ersichtlich ist, entsteht Wachstum erst, nachdem der akute Einbruch an psychischer Funktionalität bewältigt wurde. Ohne therapeutische Begleitung kann davon ausgegangen werden, dass posttraumatisches Wachstum frühestens etwa eineinhalb Jahre nach dem Ereignis auftritt (Mangelsdorf et al. 2019). Als Konsequenz sollte die therapeutische Arbeit an posttraumatischem Wachstum erst beginnen, wenn die akute Phase der Anpassung bereits bewältigt wurde.

In einer 2015 erschienenen Metaanalyse fand Roepke heraus, dass posttraumatisches Wachstum aktiv unterstützt werden kann (Roepke 2015). Im Fokus der Metaanalyse standen vor allem Interventionsformate, die nicht unmittelbar dafür entwickelt wurden, Wachstum zu ermöglichen, wie beispielsweise Trainings zur Emotionsregulation und Achtsamkeit, Psychoedukation und verhaltenstherapeutische Methoden (siehe auch Zoellner et al. 2011). Die Erforschung expliziter Behandlungskonzepte zur Förderung von posttraumatischem Wachstum steht noch aus. Deshalb sollen im Folgenden zwei konkrete Ansätze zur Arbeit mit traumatisierten PatientInnen vorgestellt werden, mit dem Hinweis, dass diese teils noch nicht oder nur in ersten Ansätzen empirisch validiert wurden. Die drei oben benannten Komponenten - die Anwesenheit unterstützender Beziehungen und positiver Emotionen sowie das Finden von Sinn im Erlebten - stellen hier wichtige Wegweiser dar. Da die therapeutische Beziehung ohnehin Grundlage der gemeinsamen Arbeit ist und die Förderung anderer tragender Beziehungen und Erarbeitung neuer Rollen zum etablierten therapeutischen Repertoire gehört, soll hier vor allem auf die anderen beiden Förderungsmöglichkeiten eingegangen werden.

\subsection{Unterstuitzung positiver Emotionen}

Die Verarbeitung potenziell traumatischer Erfahrungen ist vor allem durch eines geprägt: das Erleben von Leid. Die damit verbundenen negativen Emotionen von Angst, Wut, Trauer, Verzweiflung, Scham und Schuld, die ein wichtiger Teil des Verarbeitungsprozesses sind, verengen auch die kognitiven Möglichkeiten, neue Per- 
spektiven und Denkmuster für die Integration zu nutzen. Umgekehrt ermöglichen Momente positiver Emotionen, dass neue Lösungswege gefunden und neue Perspektiven eingenommen werden können (Fredrickson 2004, S. 1367). Sie erleichtern den Aufbau neuer Beziehungen und dämpfen die Wirkung negativer Emotionen (Fredrickson et al. 2000, S. 237). In einer prospektiven Längsschnittstudie, die Resilienz und die Entstehung von posttraumatischem Wachstum in Folge der terroristischen Angriffe auf das World Trade Center untersuchte, zeigte sich, dass die Anwesenheit positiver Emotionen die Entstehung posttraumatischen Wachstums vorhersagte (Fredrickson et al. 2003, S. 365).

Während es bislang kaum kontrollierte Interventionsstudien dazu gibt, legen diese Erkenntnisse nahe, dass die gezielte Förderung positiver Emotionen bei der Traumabearbeitung hilfreich sein kann. Hier sollen in aller Kürze drei niedrigschwellige Methoden geschildert werden, mit denen positive Emotionen unterstützt werden können.

Drei gute Dinge Für diese empirisch belegte Übung werden die KlientInnen gebeten, am Ende jeden Tages drei Dinge niederzuschreiben, für die sie heute dankbar sind (Emmons and McCullough 2003). Diese Übung bewirkt einen Perspektivwechsel und eine Aufmerksamkeitsverschiebung hin zu den positiven Aspekten des eigenen Lebens.

4-Evening-Questions In einer tiefergehenden Form der oben beschriebenen Übung, deren Wirkung ebenfalls belegt werden konnte (Ebner 2017), werden für den Tagesrückblick vier verschiedene Fragen beantwortet: 1. Was hat mir heute Freude bereitet? 2. Wofür und wem kann ich heute dankbar sein? 3. Wo habe ich mich heute lebendig gefühlt? 4. Welche Stärken konnte ich heute ausleben? Während die ersten beiden Fragen an die Übung der „Drei guten Dinge“ anschließen, zielen Frage 3 und 4 darauf $a b$, Klientinnen und Klienten ihren eigenen Stärken näher zu bringen. Besonders die gemeinsame Reflexion der über einen längeren Zeitraum gesammelten Antworten auf Frage 3 und 4 können entscheidende Momente sein, um Ressourcen für die erfolgreiche Bewältigung der traumatischen Erfahrung zu erschließen. Nachdem zentrale Stärken identifiziert wurden, kann eine bedeutsame Anschlussfrage sein: Wie können Sie diese Stärke bei der Bewältigung der aktuellen Herausforderung konkret nutzen?

Miniurlaub Eine weitere bewährte Methode, um positive Emotionen zu fördern, ist die konkrete Planung positiver Aktivitäten. Hierzu erstellen die KlientInnen eine möglichst umfangreiche Liste von Aktivitäten, die sie als wohltuend und beglückend erleben. Anschließend geht es darum, ein tägliches Zeitfenster von 20 Minuten für einen „Miniurlaub“ fest zu reservieren, in dem mindestens eine dieser Aktivitäten realisiert wird.

Die Unterstützung positiver Emotionen durch diese und andere Übungen kann ein entscheidender Schlüssel sein, um die psycho-emotionalen Grundlagen für die Förderung posttraumatischen Wachstums zu schaffen. 


\subsection{Das Finden von Sinn im Erlebten}

Eine von Betroffenen häufig formulierte Frage in der Bewältigungsphase ist: „Warum gerade ich?“ Oft wird diese Frage missverstanden als der Versuch, das Vergangene ungeschehen zu machen oder als Wunsch, dass dieses Schicksal doch jemand anderem hätte zufallen können. Viel häufiger ist sie aber der Versuch, Sinn zu finden in einer als schmerzvoll und zugleich sinnlos erlebten Situation. Dies kann therapeutisch genutzt werden.

Einen ersten Hinweis darauf, wie dies geschehen könnte, gibt eine 2008 veröffentlichte Forschungsarbeit von Watkins und Kollegen (Watkins et al. 2008). In einer wegbereitenden Interventionsstudie ließen sie die ProbandInnen ihre kritischen Erfahrungen aus drei verschiedenen Perspektiven beschreiben. Die erste Gruppe bekam die Anweisung, nur konkret über das zu schreiben, was passiert war. In der zweiten Gruppe schrieben die ProbandInnen über das Erlebte mit dem Fokus darauf, welche Emotionen es bei ihnen hervorgerufen hatte, und die letzte Gruppe wurde gebeten, das Erlebte aus einer Perspektive der Dankbarkeit zu schildern. Es stellte sich heraus, dass das Schreiben mit dem Fokus auf die erlebten Emotionen einen stärkeren Effekt hatte als das reine Berichten des Erlebten, dass aber die Perspektive der Dankbarkeit die positivste Wirkung auf die ProbandInnen hatte (Watkins et al. 2008). Es ist wichtig anzumerken, dass sich Watkins Studie mit unangenehmen, nicht aber explizit mit traumatischen Erinnerungen beschäftigte. Trotzdem kann dieses Herangehen ein wichtiger Wegweiser für die Unterstützung posttraumatischen Wachstums sein und eine Möglichkeit darstellen, Sinn aus dem Erlebten zu schöpfen.

Da die Perspektive der Dankbarkeit nur sehr sensibel im Traumakontext einzubringen ist, und sich grundsätzlich nur auf das durch die Bewältigung Erlernte, nicht aber das Trauma selbst beziehen sollte, eignen sich für die Arbeit mit traumatisierten Personen vor allem Leitfragen wie: 1) Aus heutiger Sicht: Wofür war es gut? 2) Wie können Sie die Erfahrungen, die Sie gesammelt haben, heute für sich oder andere nutzbar machen? 3) Wozu hat Sie die Erfahrung befähigt? Die schriftliche oder mündliche Beantwortung dieser Fragen kann bei Betroffenen einen Perspektivwechsel hervorrufen, der die Integration erleichtern und Wachstum positiv beeinflussen kann. Besonders Frage 2 sollte hierbei im Mittelpunkt stehen und konkret in praktisches Tun übersetzt werden.

\section{Kritik und Herausforderungen - Das Janusgesicht des posttraumatischen Wachstums}

Der römische Gott Janus hat zwei Gesichter. Eines schaut nach vorn, das andere nach hinten. Abgeleitet von dieser mythischen Gestalt ist das Janusgesicht von jeher Symbol für Zwiespältigkeit, die nicht zuletzt auch Teil posttraumatischen Wachstums ist (Maercker und Zoellner 2004, S. 41). Für viele Menschen sind die traumatischen Ereignisse der Vergangenheit mit großen Verlusten verbunden, die nicht selten auch die Gegenwart noch mitbestimmen. So stellt die Aussicht eines Gewinns im erlebten Leid eine Verlockung und auch einen Wunsch dar, dem ansonsten als sinnlos erlebten Geschehen etwas Wertvolles beizumessen. Die meisten Menschen neigen dazu, 
bereitwillig positive Konsequenzen traumatischer Erfahrungen anzunehmen, wenn sie direkt gefragt werden (Jayawickreme und Blackie 2014, S. 320). Studien, in denen untersucht wurde, ob die retrospektive Selbstwahrnehmung von Wachstum den tatsächlichen Veränderungsprozessen entspricht (Frazier et al. 2009; Ransom 2005), kamen zu dem Schluss, dass selbstwahrgenommene Veränderung nur in geringem Maße mit echtem psychologischen Wachstum zusammenhängt. Daher ist davon auszugehen, dass nur ein Teil der wahrgenommenen Veränderungsprozesse tatsächlich psychologische Reifung darstellt, während es sich bei dem anderen Teil um positive Illusionen handelt (Taylor und Armor 1996, S. 873).

Neben der Doppeldeutigkeit von Selbstaussagen über posttraumatisches Wachstum, die vor allem ein empirisches Problem darstellt, besteht eine der größten Herausforderungen in dem möglichen Aufforderungscharakter für Traumatisierte, Wachstum zeigen zu müssen. Die Gefahr besteht darin, dass durch die Betonung der Möglichkeit posttraumatisches Wachstum zu erleben, der gesellschaftliche und persönliche Druck erhöht wird, ein Trauma positiv zu bewältigen. Dies kann zu einer zusätzlichen Belastung für Betroffene werden. Gerade deshalb kann es hilfreich sein, sehr achtsam mit psychoedukativen Maßnahmen zu dem Thema umzugehen und stattdessen therapeutisch Faktoren zu stützen, die Wachstum ermöglichen, ohne es verbal zu explizieren.

\section{Fazit}

Kritische und traumatische Ereignisse werden bis heute vor allem als psycho-emotionale Bedrohung verstanden (siehe Filipp und Aymann 2018). Traumatherapeutische Arbeit orientierte sich daher primär daran, die negativen Folgen von Traumatisierungen aufzulösen (Huber 2012). Erst in der jüngeren Traumaforschung, die hier vorgestellt wurde, wird dieses Herangehen durch die Möglichkeit ergänzt, dass potenziell traumatische Ereignisse auch Katalysatoren für psychische Reifung und persönliches Wachstum sein können. Während die prospektive Wirksamkeitsforschung von unterstützenden Faktoren für posttraumatisches Wachstum noch immer in den Kinderschuhen steckt, ergeben sich erste vielversprechende Ansätze, wie posttraumatisches Wachstum therapeutisch unterstützt und gefördert werden kann. Durch diese veränderte Perspektive auf traumatische Erfahrungen können sie nicht nur als schmerzlich, sondern auch wegweisend verstanden werden und damit auch Wegbereiter sein in eine neue, erfülltere Zukunft.

Funding Open Access funding provided by Projekt DEAL.

Open Access Dieser Artikel wird unter der Creative Commons Namensnennung 4.0 International Lizenz veröffentlicht, welche die Nutzung, Vervielfältigung, Bearbeitung, Verbreitung und Wiedergabe in jeglichem Medium und Format erlaubt, sofern Sie den/die ursprünglichen Autor(en) und die Quelle ordnungsgemäß nennen, einen Link zur Creative Commons Lizenz beifügen und angeben, ob Änderungen vorgenommen wurden.

Die in diesem Artikel enthaltenen Bilder und sonstiges Drittmaterial unterliegen ebenfalls der genannten Creative Commons Lizenz, sofern sich aus der Abbildungslegende nichts anderes ergibt. Sofern das betreffende Material nicht unter der genannten Creative Commons Lizenz steht und die betreffende Handlung nicht nach gesetzlichen Vorschriften erlaubt ist, ist für die oben aufgeführten Weiterverwendungen des Materials die Einwilligung des jeweiligen Rechteinhabers einzuholen. 
Weitere Details zur Lizenz entnehmen Sie bitte der Lizenzinformation auf http://creativecommons.org/ licenses/by/4.0/deed.de.

\section{Literatur}

Bonanno, G. A. (2004). Loss, trauma, and human resilience: have we underestimated the human capacity to thrive after extremely aversive events? American Psychologist, 59, $20-28$.

Cann, A., Calhoun, L. G., Tedeschi, R. G., Kilmer, R.P., Gil-Rivas, V., Vishnevsky, T., \& Danhauer, S.C. (2010). The core beliefs inventory: a brief measure of disruption in the assumptive world. Anxiety, Stress, and Coping, 23, 19-34. https://doi.org/10.1080/10615800802573013.

Cicchetti, D., \& Toth, S.L. (2009). The past achievements and future promises of developmental psychopathology: the coming of age of a discipline. Journal of Child Psychology and Psychiatry, 50, 16-25. https://doi.org/10.1111/j.1469-7610.2008.01979.x.

Ebner, M. (2017). 4-Evening-Questions: Eine einfache Technik mit tiefgreifender Wirkung. Organisationsberatung, Supervision, Coaching, 24, 269-282.

Emmons, R. A., \& McCullough, M.E. (2003). Counting blessings versus burdens: An experimental investigation of gratitude and subjective well-being in daily life. Journal of Personality and Social Psychology, 84, 377-389.

Filipp, S.H., \& Aymanns, P. (2018). Kritische Lebensereignisse und Lebenskrisen: Vom Umgang mit den Schattenseiten des Lebens. Stuttgart: Kohlhammer.

Frazier, P., Tennen, H., Gavian, M., Park, C., Tomich, P., \& Tashiro, T. (2009). Does self-reported posttraumatic growth reflect genuine positive change? Psychological Science, 20, 912-919. https://doi.org/ 10.1111/j.1467-9280.2009.02381.x.

Fredrickson, B.L. (2004). The broaden-and-build theory of positive emotions. Philosophical Transactions of the Royal Society of London. Series B, Biological Sciences, 359, 1367-1378. https://doi.org/10. 1098/rstb.2004.1512.

Fredrickson, B.L., Mancuso, R. A., Branigan, C., \& Tugade, M. M. (2000). The undoing effect of positive emotions. Motivation and Emotion, 24, 237-258.

Fredrickson, B.L., Tugade, M., Waugh, C.E., \& Larkin, G. R. (2003). What good are positive emotions in crises? A prospective study of resilience and emotions following the terrorist attacks on the United States on September 11th, 2001. Journal of Personality and Social Psychology, 84, 365-376. https:// doi.org/10.1037/0022-3514.84.2.365.

Huber, M. (2012). Trauma und die Folgen: Trauma und Traumabehandlung. Paderborn: Junfermann.

Jayawickreme, E., \& Blackie, L. (2014). Posttraumatic growth as positive personality change: Evidence, controversies and future directions. European Journal of Personality, 28, 312-331. https://doi.org/10. 1002/per. 1963.

Jayawickreme, E., \& Blackie, L.E.R. (2016). Exploring the psychological benefits of hardship: A critical reassessment of posttraumatic growth. New York: Springer.

Joseph, S., \& Linley, P. A. (2004). Adversarial growth and positive change following trauma: Theory, research, and practice. Ricerche di Psicologia, 27, 177-190.

Maercker, A., \& Zoellner, T. (2004). The Janus face of self-perceived growth: Toward a two-component model of posttraumatic growth. Psychological Inquiry, 15, 41-48. https://doi.org/10.2307/20447200.

Mangelsdorf, J. (2014). Posttraumatic and postecstatic growth in medicine. In M. W. Snyder (Hrsg.), Positive Health (S. 208-222). Bloomington: Balboa Press.

Mangelsdorf, J. (2017). Coping with childbirth: Brain structural associations of personal growth initiative. Frontiers in Psychology, 8, 1-9.

Mangelsdorf, J., \& Eid, M. (2015). What makes a thriver? Unifying the concepts of posttraumatic and postecstatic growth. Frontiers in Psychology, 6, 1-17.

Mangelsdorf, J., Eid, M., \& Luhmann, M. (2019). Does growth require suffering? A systematic review and meta-analysis on genuine posttraumatic and postecstatic growth. Psychological Bulletin. https://doi. org/10.1037/bul0000173.

Masten, A. S. (2014). Invited commentary: resilience and positive youth development frameworks in developmental science. Journal of Youth and Adolescence, 43, 1018-1024. https://doi.org/10.1007/ s10964-014-0118-7.

Nietzsche, F. (1997). Twilight of the Idols. Indianapolis: Hackett. 
Overton, W.F. (2015). Processes, relations and relational-developmental systems. In W.F. Overton \& P.C.M. Molenar (Hrsg.), Theory and method: Handbook of child psychology and developmental science (S. 9-62). Hoboken: Wiley. https://doi.org/10.1002/9781118963418.childpsy102.

Park, C. L., \& Helgeson, V.S. (2006). Introduction to the special section: growth following highly stressful life events: current status and future directions. Journal of Consulting and Clinical Psychology, 74, 791-796. https://doi.org/10.1037/0022-006X.74.5.791.

Park, C.L., Cohen, L.H., \& Murch, R.L. (1996). Assessment and prediction of stress-related growth. Journal of Personality, 64, 71-105.

Rabe, S., Zöllner, T., Maercker, A., \& Karl, A. (2006). Neural correlates of posttraumatic growth after severe motor vehicle accidents. Journal of Consulting and Clinical Psychology, 74, 880-886. https:// doi.org/10.1037/0022-006X.74.5.880.

Ransom, S. (2005). Temporal comparisons and the perception of posttraumatic growth in early stage cancer patients (Promotion). http://scholarcommons.usf.edu/etd/2955. Zugegriffen: 29.05.2019.

Roepke, A. M. (2015). Psychosocial interventions and posttraumatic growth: A meta-analysis. Journal of Consulting and Clinical Psychology, 83, 129-142.

Shapiro, F. (2017). Eye movement desensitization and reprocessing (EMDR) therapy: basic principles, protocols, and procedures. New York: Guilford.

Spangenberg, E. (2015). Behutsame Trauma-Integration (TRIMB): Belastende Erfahrungen lösen mit Atmung, Bewegung und Imagination. Stuttgart: Klett-Cotta.

Taylor, S.E., \& Armor, D. A. (1996). Positive illusions and coping with trauma. Journal of Personality, 64, 873-898. https://doi.org/10.1111/j.1467-6494.1996.tb00947.x.

Tedeschi, R.G., \& Calhoun, L.G. (1996). The posttraumatic growth inventory: measuring the positive legacy of trauma. Journal of Traumatic Stress, 9, 455-471. https://doi.org/10.1002/jts.2490090305.

Tedeschi, R.G., \& Calhoun, L.G. (2004). Posttraumatic growth: conceptual foundations and empirical evidence. Psychological Inquiry, 15, 1-18. https://doi.org/10.1207/s15327965pli1501_01.

Watkins, P.C., Cruz, L., Holben, H., \& Kolts, R. L. (2008). Taking care of business? Grateful processing of unpleasant memories. The Journal of Positive Psychology, 3, 87-99. https://doi.org/10.1080/ 17439760701760567.

Zoellner, T., Rabe, S., Karl, A., \& Maercker, A. (2011). Post-traumatic growth as outcome of a cognitivebehavioural therapy trial for motor vehicle accident survivors with PTSD. Psychology and Psychotherapy: Theory, Research and Practice, 84(2), 201-213.

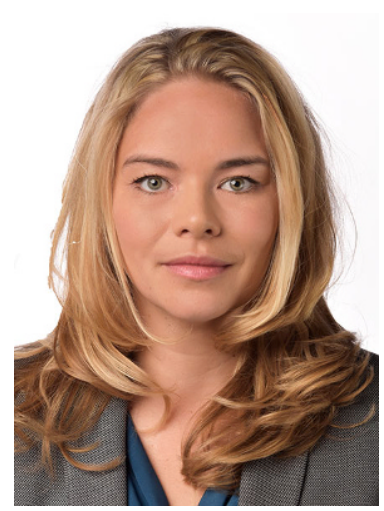

Dr. Judith Mangelsdorf 1984, Dr., Dipl.-Psych., MEd., MAPP, Institutsleiterin der Deutsche Gesellschaft für Positive Psychologie, Supervisorin, 2. Vorsitzende des Deutschsprachigen Dachverbandes für Positive Psychologie e. V. 